\title{
A NOTE ON PERIODIC SOLUTIONS IN THE VICINITY OF A CENTER
}

\author{
BAU-SEN DU
}

\begin{abstract}
We show that a small polynomial perturbation of a center will result in periodic solutions provided the coefficients of the polynomial satisfy inequality (2) below. The proof is based on an application of degree theory.
\end{abstract}

1. Introduction. In her book [1] (see also [2]), Cronin stated the following. Let $h_{1}(x, y, \mu)$ and $h_{2}(x, y, \mu)$ be polynomials in $x, y$ and $\mu$ and let $k_{1}(\sin \theta, \cos \theta, \mu)$, $k_{2}(\sin \theta, \cos \theta, \mu)$ be polynomials in $\sin \theta, \cos \theta$ and $\mu$. Assume that $\eta(\mu)$ is a $C^{1}$ function and $\eta_{0}=\eta(0) \neq 0$. Let $\omega=[1+\mu \eta(\mu)]^{-1}$ and $\tau=\omega t$. Then the following two-dimensional nonlinear differential system

$$
\left\{\begin{array}{l}
x^{\prime}=y+\mu\left[h_{1}(x, y, \mu)+k_{1}(\sin \tau, \cos \tau, \mu)\right], \\
y^{\prime}=-x+\mu\left[h_{2}(x, y, \mu)+k_{2}(\sin \tau, \cos \tau, \mu)\right], \quad\left({ }^{\prime}=\frac{d}{d t}\right),
\end{array}\right.
$$

has, for all sufficiently small $\mu$, at least one solution of period $2 \pi(1+\mu \eta(\mu))$.

For the proof of this result, Dr. Cronin used the results of degree theory, in particular, the homotopy theorem and the odd mapping theorem as applied to a function $N_{\mu}(C)$ which depends on the terms $h_{1}, h_{2}, k_{1}$ and $k_{2}$. The quantity $C$ represents the initial data for the solution which is periodic precisely when $N_{\mu}(C)=0$. But it seems that, in some cases, one cannot apply the odd mapping theorem. For example, if we let $\eta(\mu) \equiv 1, \omega=(1+\mu)^{-1}, \tau=\omega \tau$ and consider

$$
\left\{\begin{array}{l}
x^{\prime}=y+\mu(x+3 y+\cos \tau), \\
y^{\prime}=-x+\mu(x-y-\sin \tau),
\end{array}\right.
$$

then $N_{0}(C) \equiv\left(\begin{array}{c}2 \pi \\ 0\end{array}\right)$. (From now on, we shall use the same notation as Cronin [1, pp. 91-93].) So the degree of $N_{0}(C)$ in any domain is 0 . In this case, we cannot conclude from degree theory that the differential system (*) has, for all sufficiently small $\mu$, at least one solution of period $2 \pi(1+\mu)$. The purpose of this paper is to compute directly $N_{0}(C)$. We will show that if the coefficients of $h_{1}$ and $h_{2}$ satisfy a simple inequality, then the differential system (1) has a periodic solution for $\mu$ sufficiently small. The precise statement of our result is given in the next section, but we note that the inequality required is a generic condition on $h_{1}$ and $h_{2}$, and consequently most systems satisfy the conclusion of Cronin's Theorem. The proof of our result appears in $\$ \S 3$ and 4.

Received by the editors March 6, 1980.

1980 Mathematics Subject Classification. Primary 34C25.

(c) 1981 American Mathematical Society 0002-9939/81/0000-0167/\$02.50 
2. Statement of the theorem. For nonnegative integers $\boldsymbol{n}$ and $k$, we define

$$
\alpha_{k, n}= \begin{cases}\sum_{j=0}^{k}(-1)^{k-j}\left(\begin{array}{c}
k \\
j
\end{array}\right)\left(\begin{array}{c}
2 n+1-k \\
n-j
\end{array}\right) & \text { if } 0<k<n, \\
\sum_{j=0}^{2 n+1-k}(-1)^{k+j-n}\left(\begin{array}{c}
k \\
n-j
\end{array}\right)\left(\begin{array}{c}
2 n+1-k \\
j
\end{array}\right) & \text { if } n+1<k<2 n+1 .\end{cases}
$$

Then, it is not hard to show that

$$
\begin{aligned}
\alpha_{k, n} & =\frac{4^{n}}{\pi i^{k}} \int_{0}^{2 \pi} \cos ^{2 n+1-k} \theta \cdot(-1)^{k} \sin ^{k} \theta \cdot e^{i \theta} d \theta \\
& =\left\{\begin{array}{l}
\frac{4^{n}}{\pi} \cdot 2 \pi \cdot \frac{1 \cdot 3 \cdot 5 \cdots(2 n+1)}{2 \cdot 4 \cdot 6 \cdot \cdots(2 n+2)}, \quad \text { if } k=0, \\
\frac{4^{n} \cdot i^{k}}{\pi} \cdot 2 \pi \cdot \frac{1 \cdot 3 \cdot 5 \cdots(2 n+1-k) \cdot 1 \cdot 3 \cdot 5 \cdots(k-1)}{2 \cdot 4 \cdot 6 \cdot(2 n+2)} \\
\text { if } k \text { is even and } 0<k<2 n+1, \\
\frac{4^{n} \cdot i^{k+1}}{\pi} \cdot 2 \pi \cdot \frac{1 \cdot 3 \cdot 5 \cdots(2 n-k) \cdot 1 \cdot 3 \cdot 5 \cdots k}{2 \cdot 4 \cdot 6 \cdot \cdots(2 n+2)} \\
\text { if } k \text { is odd and } 0<k<2 n+1, \\
\frac{(-1)^{n+1} \cdot 4^{n}}{\pi} \cdot 2 \pi \cdot \frac{1 \cdot 3 \cdot 5 \cdots(2 n+1)}{2 \cdot 4 \cdot 6 \cdots(2 n+2)}, \quad \text { if } k=2 n+1 .
\end{array}\right.
\end{aligned}
$$

Let

$$
\begin{aligned}
& P_{n}(x, y)=\sum_{k=0}^{2 n+1} a_{k, n} x^{2 n+1-k} y^{k} \\
& Q_{n}(x, y)=\sum_{k=0}^{2 n+1} b_{k, n} x^{2 n+1-k} y^{k}
\end{aligned}
$$

and let

$$
\begin{aligned}
& A_{n}=\sum_{k=0}^{n}(-1)^{k}\left(a_{2 k, n} \alpha_{2 k, n}-b_{2 k+1, n} \alpha_{2 k+1, n}\right), \\
& B_{n}=\sum_{k=0}^{n}(-1)^{k}\left(a_{2 k+1, n} \alpha_{2 k+1, n}+b_{2 k, n} \alpha_{2 k, n}\right) .
\end{aligned}
$$

Now we can state the following theorem.

Theorem. Assume that $h_{1}(x, y, \mu)$ and $h_{2}(x, y, \mu)$ are polynomials in $x, y$ and $\mu$. When $\mu=0$, let

$$
\begin{aligned}
& h_{1}(x, y, 0)=P(x, y)+\sum_{n=0}^{N} P_{n}(x, y), \\
& h_{2}(x, y, 0)=Q(x, y)+\sum_{n=0}^{N} Q_{n}(x, y),
\end{aligned}
$$


where $P(x, y), Q(x, y)$ are polynomials in $x, y$ in which each term is of even degree. Define $A_{n}, B_{n}$ as above, and let $k_{1}(\sin \theta, \cos \theta, \mu)$ and $k_{2}(\sin \theta, \cos \theta, \mu)$ be polynomials in $\sin \theta, \cos \theta$ and $\mu$. Assume that $\eta(\mu)$ is a $C^{1}$ function and $\eta_{0}=\eta(0)$. Let $\omega=[1+\mu \eta(\mu)]^{-1}$ and $\tau=\omega t$. If

$$
\left(a_{0,0}+b_{1,0}\right)^{2}+\left(2 \eta_{0}-a_{1,0}+b_{0,0}\right)^{2}+\sum_{n=1}^{N}\left(A_{n}^{2}+B_{n}^{2}\right) \neq 0
$$

then the following two-dimensional nonlinear differential system

$$
\left\{\begin{array}{l}
x^{\prime}=y+\mu\left\{h_{1}(x, y, \mu)+k_{1}(\sin \tau, \cos \tau, \mu)\right\} \\
y^{\prime}=-x+\mu\left\{h_{2}(x, y, \mu)+k_{2}(\sin \tau, \cos \tau, \mu)\right\}
\end{array}\right.
$$

has, for all sufficiently small $|\mu|$, at least one periodic solution of period $2 \pi(1+\mu \eta(\mu))$.

COROLlaRY. If, in the above theorem, we let

$$
\begin{aligned}
& h_{1}(x, y, 0)=a_{0}+a_{1} x+a_{2} y+H . O . T ., \\
& h_{2}(x, y, 0)=b_{0}+b_{1} x+b_{2} y+H . O . T .,
\end{aligned}
$$

and assume $\left(a_{1}+b_{2}\right)^{2}+\left(2 \eta_{0}-a_{2}+b_{1}\right)^{2} \neq 0$, then the conclusion of the above theorem holds.

REMARK. We call attention to the fact that this theory applies in cases where $\eta_{0}=0$.

3. Computation of $N_{0}(C)$. Let

$$
C=\left(\begin{array}{l}
c_{1} \\
c_{2}
\end{array}\right)
$$

and let $z=c_{1}-i c_{2}$. When $\mu=0$, we let

$$
\left(\begin{array}{l}
x \\
y
\end{array}\right)=\left(\begin{array}{l}
x(\theta, 0, C) \\
y(\theta, 0, C)
\end{array}\right)=\left(\begin{array}{c}
c_{1} \cos \theta+c_{2} \sin \theta \\
-c_{1} \sin \theta+c_{2} \cos \theta
\end{array}\right)=\left(\begin{array}{l}
\frac{1}{2}\left(e^{i \theta_{z}}+e^{-i \theta} \bar{z}\right) \\
\frac{i}{2}\left(e^{i \theta_{z}}-e^{-i \theta} \bar{z}\right)
\end{array}\right) .
$$

In particular, when $z=1$, one has $x=\cos \theta, y=-\sin \theta$.

In Cronin's notation, one has

$$
N_{0}(C)=\left(\begin{array}{c}
2 \pi \eta_{0} c_{2}+H_{1}\left(c_{1}, c_{2}\right)+K_{1} \\
-2 \pi \eta_{0} c_{1}+H_{2}\left(c_{1}, c_{2}\right)+K_{2}
\end{array}\right)
$$

where

$$
\begin{aligned}
\left(\begin{array}{l}
H_{1}\left(c_{1}, c_{2}\right) \\
H_{2}\left(c_{1}, c_{2}\right)
\end{array}\right) & =\int_{0}^{2 \pi}\left(\begin{array}{l}
h_{1}(x, y, 0) \cos \theta-h_{2}(x, y, 0) \sin \theta \\
h_{1}(x, y, 0) \sin \theta+h_{2}(x, y, 0) \cos \theta
\end{array}\right) d \theta \\
& =\int_{0}^{2 \pi}\left(\begin{array}{l}
\operatorname{Re}\left\{\left[h_{1}(x, y, 0)+i h_{2}(x, y, 0)\right] e^{i \theta}\right\} \\
\operatorname{Im}\left\{\left[h_{1}(x, y, 0)+i h_{2}(x, y, 0)\right] e^{i \theta}\right\}
\end{array}\right) d \theta
\end{aligned}
$$


582

BAU-SEN DU

and

$$
\left(\begin{array}{l}
K_{1} \\
K_{2}
\end{array}\right)=\int_{0}^{2 \pi}\left(\begin{array}{l}
k_{1}(\sin \theta, \cos \theta, 0) \cos \theta-k_{2}(\sin \theta, \cos \theta, 0) \sin \theta \\
k_{1}(\sin \theta, \cos \theta, 0) \sin \theta+k_{2}(\sin \theta, \cos \theta, 0) \cos \theta
\end{array}\right) d \theta
$$

is a constant vector.

Since $h_{1}, h_{2}$ are polynomials in $x$ and $y$, it suffices to compute $\int_{0}^{2 \pi} h(x, y) e^{i \theta} d \theta$, where $h(x, y)$ is a polynomial in $x$ and $y$.

Given two nonnegative integers $m$ and $n$, and let $h(x, y)=x^{m} y^{n}$. If $m+n$ is even, then it is trivial that $\int_{0}^{2 \pi} h(x, y) e^{i \theta} d \theta=0$. So we can assume that $m+n$ is odd.

If $h(x, y)=x^{2 n+1-k} y^{k}$, where $0<k<n$, then

$$
\begin{aligned}
\int_{0}^{2 \pi} h(x, y) e^{i \theta} d \theta & =\int_{0}^{2 \pi} x^{2 n+1-k} y^{k} e^{i \theta} d \theta \\
& =\int_{0}^{2 \pi} \frac{1}{2^{2 n+1-k}}\left(e^{i \theta} z+e^{-i \theta} \bar{z}\right)^{2 n+1-k}\left(\frac{i}{2}\right)^{k}\left(e^{i \theta} z-e^{-i \theta} \bar{z}\right)^{k} e^{i \theta} d \theta \\
& =\frac{i^{k} \cdot \pi}{4^{n}}(z \bar{z})^{n} \bar{z} \cdot \sum_{j=0}^{k}(-1)^{k-j}\left(\begin{array}{c}
k \\
j
\end{array}\right)\left(\begin{array}{c}
2 n+1-k \\
n-j
\end{array}\right) \\
& =\frac{i^{k} \pi}{4^{n}}(z \bar{z})^{n} \bar{z} \cdot \alpha_{k, n} .
\end{aligned}
$$

Similarly, if $h(x, y)=x^{2 n+1-k} y^{k}$, where $n+1<k<2 n+1$, then

$$
\begin{aligned}
\int_{0}^{2 \pi} h(x, y) e^{i \theta} d \theta & =\int_{0}^{2 \pi} x^{2 n+1-k} y^{k} e^{i \theta} d \theta \\
& =\frac{i^{k} \pi}{4^{n}}(z \bar{z})^{n} \bar{z} \cdot \sum_{j=0}^{2 n+1-k}(-1)^{k+j-n}\left(\begin{array}{c}
k \\
n-j
\end{array}\right)\left(\begin{array}{c}
2 n+1-k \\
j
\end{array}\right) \\
& =\frac{i^{k} \pi}{4^{n}}(z \bar{z})^{n} \bar{z} \cdot \alpha_{k, n} .
\end{aligned}
$$

Now assume

$$
\begin{aligned}
& h_{1}(x, y, 0)=\sum_{k=0}^{2 n+1} a_{k, n} x^{2 n+1-k_{y} k}=P_{n}(x, y), \\
& h_{2}(x, y, 0)=\sum_{k=0}^{2 n+1} b_{k, n} x^{2 n+1-k_{y} k}=Q_{n}(x, y), \quad(n>0) .
\end{aligned}
$$


Then

$$
\begin{aligned}
\int_{0}^{2 \pi}[ & \left.h_{1}(x, y, 0)+i h_{2}(x, y, 0)\right] e^{i \theta} d \theta \\
= & \sum_{k=0}^{2 n+1}\left(a_{k, n}+i b_{k, n}\right) \int_{0}^{2 \pi} x^{2 n+1-k} y^{k} e^{i \theta} d \theta \\
= & \sum_{k=0}^{2 n+1}\left(a_{k, n}+i b_{k, n}\right) \cdot \frac{i^{k} \pi}{4^{n}} \cdot(z \bar{z})^{n} \bar{z} \cdot \alpha_{k, n} \\
= & \frac{\pi}{4^{n}}(z \bar{z})^{n} \bar{z} \cdot\left[\sum_{k=0}^{n}(-1)^{k} a_{2 k, n} \alpha_{2 k, n}-\sum_{k=0}^{n}(-1)^{k} b_{2 k+1, n} \alpha_{2 k+1, n}\right] \\
& +i \frac{\pi}{4^{n}}(z \bar{z})^{n} \bar{z} \cdot\left[\sum_{k=0}^{n}(-1)^{k} a_{2 k+1, n} \alpha_{2 k+1, n}+\sum_{k=0}^{n}(-1)^{k} b_{2 k, n} \alpha_{2 k, n}\right] \\
= & \frac{\pi}{4^{n}}(z \bar{z})^{n} \bar{z} A_{n}+i \frac{\pi}{4^{n}}(z \bar{z})^{n} \bar{z} B_{n} \quad\left(\text { where } A_{n}, B_{n} \text { are as defined in } 82\right) \\
= & \frac{\pi}{4^{n}}(z \bar{z})^{n} \bar{z}\left(A_{n}+i B_{n}\right) \\
= & \frac{\pi}{4^{n}}\left(c_{1}^{2}+c_{2}^{2}\right)^{n}\left(c_{1}+i c_{2}\right)\left(A_{n}+i B_{n}\right) \\
= & \frac{\pi}{4^{n}}\left(c_{1}^{2}+c_{2}^{2}\right)^{n}\left[\left(c_{1} A_{n}-c_{2} B_{n}\right)+i\left(c_{1} B_{n}+c_{2} A_{n}\right)\right] .
\end{aligned}
$$

Therefore, the real and imaginary parts of $\int_{0}^{2 \pi}\left[P_{n}(x, y)+i Q_{n}(x, y)\right] e^{i \theta} d \theta$ are $\left(\pi / 4^{n}\right)\left(c_{1}^{2}+c_{2}^{2}\right)^{n}\left(c_{1} A_{n}-c_{2} B_{n}\right)$ and $\left(\pi / 4^{n}\right)\left(c_{1}^{2}+c_{2}^{2}\right)^{n}\left(c_{1} B_{n}+c_{2} A_{n}\right)$ respectively.

Finally, we let $h_{1}(x, y, 0)=P(x, y)+\sum_{n=0}^{N} P_{n}(x, y)$, and $h_{2}(x, y, 0)=Q(x, y)$ $+\sum_{n=0}^{N} Q_{n}(x, y)$ where $P(x, y)$ and $Q(x, y)$ are polynomials in $x, y$ in which each term is of even degree, and $P_{n}(x, y), Q_{n}(x, y)$ are defined as above. Then

$$
\int_{0}^{2 \pi}\left[h_{1}(x, y, 0)+i h_{2}(x, y, 0)\right] e^{i \theta} d \theta=\sum_{n=0}^{N} \int_{0}^{2 \pi}\left[P_{n}(x, y)+i Q_{n}(x, y)\right] e^{i \theta} d \theta \text {. }
$$

(Note that

$$
\begin{aligned}
\int_{0}^{2 \pi} P(x, y) e^{i \theta} d \theta & =0=\int_{0}^{2 \pi} Q(x, y) e^{i \theta} d \theta \\
& =\sum_{n=0}^{N} \frac{\pi}{4^{n}}\left(c_{1}^{2}+c_{2}^{2}\right)^{n}\left[\left(c_{1} A_{n}-c_{2} B_{n}\right)+i\left(c_{1} B_{n}+c_{2} A_{n}\right)\right] .
\end{aligned}
$$

So the real and imaginary parts of $\int_{0}^{2 \pi}\left[h_{1}(x, y, 0)+i h_{2}(x, y, 0)\right] e^{i \theta} d \theta$ are $\sum_{n=0}^{N}\left(\pi / 4^{n}\right)\left(c_{1}^{2}+c_{2}^{2}\right)^{n}\left(c_{1} A_{n}-c_{2} B_{n}\right)$ and $\sum_{n=0}^{N}\left(\pi / 4^{n}\right)\left(c_{1}^{2}+c_{2}^{2}\right)^{n}\left(c_{1} B_{n}+c_{2} A_{n}\right)$ respectively. Therefore,

$$
\begin{aligned}
& H_{1}\left(c_{1}, c_{2}\right)=\sum_{n=0}^{N} \frac{\pi}{4^{n}}\left(c_{1}^{2}+c_{2}^{2}\right)^{n}\left(c_{1} A_{n}-c_{2} B_{n}\right), \\
& H_{2}\left(c_{1}, c_{2}\right)=\sum_{n=0}^{N} \frac{\pi}{4^{n}}\left(c_{1}^{2}+c_{2}^{2}\right)^{n}\left(c_{1} B_{n}+c_{2} A_{n}\right),
\end{aligned}
$$

where $A_{n}, B_{n}$ are as defined in $\$ 2$. So we have got the explicit formulas for $H_{1}\left(c_{1}, c_{2}\right)$ and $H_{2}\left(c_{1}, c_{2}\right)$. 
4. Proof of the theorem. Now we have obtained the formulas for $H_{1}\left(c_{1}, c_{2}\right)$ and $H_{2}\left(c_{1}, c_{2}\right)$. So

$$
\begin{aligned}
N_{0}(C)= & \left(\begin{array}{c}
2 \pi \eta_{0} c_{2}+H_{1}\left(c_{1}, c_{2}\right)+K_{1} \\
-2 \pi \eta_{0} c_{1}+H_{2}\left(c_{1}, c_{2}\right)+K_{2}
\end{array}\right) \\
= & \left(\begin{array}{l}
2 \pi \eta_{0} c_{2}+\pi\left(c_{1} A_{0}-c_{2} B_{0}\right)+\sum_{n=1}^{N} \frac{\pi}{4^{n}}\left(c_{1}^{2}+c_{2}^{2}\right)^{n}\left(c_{1} A_{n}-c_{2} B_{n}\right)+K_{1} \\
-2 \pi \eta_{0} c_{1}+\pi\left(c_{1} B_{0}+c_{2} A_{0}\right)+\sum_{n=1}^{N} \frac{\pi}{4^{n}}\left(c_{1}^{2}+c_{2}^{2}\right)^{n}\left(c_{1} B_{n}+c_{2} A_{n}\right)+K_{2}
\end{array}\right) \\
= & \pi\left(\begin{array}{l}
c_{1}\left(a_{0,0}+b_{1,0}\right)+c_{2}\left(2 \eta_{0}+b_{0,0}-a_{1,0}\right)+\sum_{n=1}^{N} \frac{\left(c_{1}^{2}+c_{2}^{2}\right)^{n}}{4^{n}}\left(c_{1} A_{n}-c_{2} B_{n}\right) \\
c_{1}\left(-2 \eta_{0}-b_{0,0}+a_{1,0}\right)+c_{2}\left(a_{0,0}+b_{1,0}\right)+\sum_{n=1}^{N} \frac{\left(c_{1}^{2}+c_{2}^{2}\right)^{n}}{4^{n}}\left(c_{1} B_{n}+c_{2} A_{n}\right)
\end{array}\right) \\
& +\left(\begin{array}{l}
K_{1} \\
K_{2}
\end{array}\right) .
\end{aligned}
$$

If for some $n>1, A_{n}^{2}+B_{n}^{2} \neq 0$, then $N_{0}(C)$ is dominated at least by $\left(c_{1}^{2}+c_{2}^{2}\right)^{n}$. For all sufficiently large $\|C\|$, one has $N_{0}(C) \neq 0$, and we can apply the odd mapping theorem. On the other hand, if $A_{n}=B_{n}=0$ for all $1<n<N$ and if $\left(a_{0,0}+b_{1,0}\right)^{2}+\left(2 \eta_{0}+b_{0,0}-a_{1,0}\right)^{2} \neq 0$, then

$$
N_{0}(C)=\pi\left(\begin{array}{c}
c_{1}\left(a_{0,0}+b_{1,0}\right)+c_{2}\left(2 \eta_{0}+b_{0,0}-a_{1,0}\right) \\
c_{1}\left(-2 \eta_{0}-b_{0,0}+a_{1,0}\right)+c_{2}\left(a_{0,0}+b_{1,0}\right)
\end{array}\right)+\left(\begin{array}{c}
K_{1} \\
K_{2}
\end{array}\right)=0
$$

for exactly one $C$. So, again, for all sufficiently large $\|C\|$, the function $N_{0}(C)$ can never be zero and the odd mapping theorem applies too. But if $a_{0,0}+b_{1,0}=0=$ $2 \eta_{0}+b_{0,0}-a_{1,0}$ and $A_{n}=B_{n}=0$ for $1<n<N$, then the function $N_{0}(C)$ is constantly equal to $\left(\begin{array}{c}K_{1} \\ K_{2}\end{array}\right)$. So its degree is either undefined or equal to zero. Hence no conclusion can be drawn in this case. This completes the proof.

Acknowledgments. I want to thank Professor G. R. Sell for his many valuable suggestions.

\section{REFERENCES}

1. J. Cronin, Fixed points and topological degree in nonlinear analysis, Math. Surveys, no. 11, Amer. Math. Soc., Providence, R. I., 1964, pp. 91-93.

2. N. G. Lloyd, Degree theory, Cambridge Univ. Press, Cambridge and New York, 1978, pp. 132-133.

SChOOL of Mathematics, University of Minnesota, Minneapolis, MnNesota 55455 